\author{
Measurement of Fast Electron Spin Relaxation Times with Atomic \\ Resolution \\ Sebastian Loth et al. \\ Science 329, 1628 (2010); \\ DOI: $10.1126 /$ science. 1191688
}

This copy is for your personal, non-commercial use only.

If you wish to distribute this article to others, you can order high-quality copies for your colleagues, clients, or customers by clicking here.

Permission to republish or repurpose articles or portions of articles can be obtained by following the guidelines here.

The following resources related to this article are available online at www.sciencemag.org (this information is current as of June 1, 2012):

Updated information and services, including high-resolution figures, can be found in the online version of this article at:

http://www.sciencemag.org/content/329/5999/1628.full.html

Supporting Online Material can be found at:

http://www.sciencemag.org/content/suppl/2010/09/22/329.5999.1628.DC1.html

A list of selected additional articles on the Science Web sites related to this article can be found at:

http://www.sciencemag.org/content/329/5999/1628.full.html\#related

This article cites 26 articles, 6 of which can be accessed free:

http://www.sciencemag.org/content/329/5999/1628.full.html\#ref-list-1

This article has been cited by 3 articles hosted by HighWire Press; see:

http://www.sciencemag.org/content/329/5999/1628.full.html\#related-urls

This article appears in the following subject collections:

Physics

http://www.sciencemag.org/cgi/collection/physics 


\title{
Measurement of Fast Electron Spin Relaxation Times with Atomic Resolution
}

\author{
Sebastian Loth, ${ }^{1 *}$ Markus Etzkorn, ${ }^{2}$ Christopher P. Lutz, ${ }^{1}$ D. M. Eigler, ${ }^{1}$ Andreas ]. Heinrich ${ }^{1 *}$
}

Single spins in solid-state systems are often considered prime candidates for the storage of quantum information, and their interaction with the environment the main limiting factor for the realization of such schemes. The lifetime of an excited spin state is a sensitive measure of this interaction, but extending the spatial resolution of spin relaxation measurements to the atomic scale has been a challenge. We show how a scanning tunneling microscope can measure electron spin relaxation times of individual atoms adsorbed on a surface using an all-electronic pump-probe measurement scheme. The spin relaxation times of individual $\mathrm{Fe}-\mathrm{Cu}$ dimers were found to vary between 50 and 250 nanoseconds. Our method can in principle be generalized to monitor the temporal evolution of other dynamical systems.

$\mathrm{W}$ hen a magnetic atom is placed in a solid or onto a surface, it can exchange energy and angular momentum with the local environment, giving a finite lifetime to its excited spin states. For non-itinerant (localized) electron systems, these interactions occur on the atomic length scale and on a time scale generally in the pico- to microsecond range. Magnetic resonance techniques are widely used to measure spin relaxation times (1), but achieving high spatial resolution of individual spins rather than ensembles has remained a challenge (2-4). Spinpolarized scanning tunneling microscopy (STM), by contrast, can probe static magnetic properties on the atomic scale, such as complex magnetic order (5), $g$-values (6), anisotropy $(7,8)$ and exchange energies $(9,10)$. However, the bandwidth of conventional STM current amplifiers (11) is insufficient to directly access typical spin relaxation times. Efforts to enhance the STM's temporal resolution through faster current amplifiers (12) or pulsed laser techniques $(13,14)$ have so far lacked atomic spatial resolution.

Here we show that the combination of an allelectronic pump-probe scheme with a spin-sensitive contrast mechanism $(15,16)$ allows the STM to measure electron spin relaxation times of individual atoms with nanosecond time resolution. In this pump-probe scheme (Fig. 1), we apply a strong voltage pulse (the pump pulse) across the tunnel junction to create spin excitations of the surface atom and a weaker voltage pulse (the probe pulse) to interrogate the state of the atom's spin at a time $\Delta t$ after the pump pulse (17). Integrating the probe-pulse current over many pump-probe cycles and slowly varying $\Delta t$ allows us to map out the average dynamical evolution of the atom's spin.

${ }^{1}$ IBM Research Division, Almaden Research Center, 650 Harry Road, San Jose, CA 95120, USA. ${ }^{2}$ Institut de Physique de la Matière Condensée, Ecole Polytechnique Fédérale de Lausanne, CH-1015 Lausanne, Switzerland.

*To whom correspondence should be addressed. E-mail: lothseb@us.ibm.com (S.L.); heinrich@almaden.ibm.com (A.].H.)
Atomic-scale structures having long spin relaxation times may have applications in information storage or quantum information processing (18). It was shown that large easy-axis magnetic anisotropy can lead to long spin relaxation times (19) that are sufficient for coherent spin manipulation (20). Fe atoms placed on a $\mathrm{Cu}_{2} \mathrm{~N}$ overlayer on a $\mathrm{Cu}(100)$ surface have shown considerable magneto-crystalline anisotropy $(8) . \mathrm{Cu}_{2} \mathrm{~N}$ binds the $\mathrm{Fe}$ atoms in a polar-covalent network that enables large anisotropy and decouples the adatoms from the $\mathrm{Cu}$ conduction electrons $(10,21,22)$, allowing Fe to retain its free atom spin of $S=2$ (8). Here we increased the easy-axis anisotropy of an $\mathrm{Fe}$ atom by placing it adjacent to a $\mathrm{Cu}$ adatom (Fig. 2A). We probed the dimer using spin-excitation spectroscopy (Fig. 2B) and found the first excitation at a threshold voltage of $V_{\mathrm{thr}}=$ $16.7 \mathrm{mV}$, a factor of 4 higher than for the individual $\mathrm{Fe}$ atom. This indicates an unusually large easy-axis anisotropy, and indeed we find spin relaxation times that exceed $200 \mathrm{~ns}$.

Spin excitations of the surface atom are driven by inelastic scattering of the tunneling electrons (O). In this process, the pump voltage must exceed the threshold voltage, $V_{\text {thr }}$, required to drive the atom's spin from the ground state to an excited state. In general, the magnetic orientation of an excited spin state will be different from that of the ground state. We can sense the orientation of the surface atom's spin by magneto-resistive tunneling with a spin-polarized tip (Fig. 1) (17). The tunnel current that flows during the probe pulse then depends on the projection of the surface atom's spin along the spin-polarization axis of the tip.

A pump-probe measurement of an $\mathrm{Fe}-\mathrm{Cu}$ dimer at $0.6 \mathrm{~K}$ temperature and $7 \mathrm{~T}$ magnetic field is shown in Fig. 2C. In our experiments, we record the probe-pulse current, which can be expressed as the number of electrons per probe pulse, $N$. We plot $\Delta N=N(\Delta t)-N(-600 \mathrm{~ns})$, the change in $N$ with respect to its value when the probe pulse precedes the pump pulse, a condition in which the spin is in its ground state during the probe pulse. The behavior of $\Delta N$ for positive delay times reveals the dynamical evolution of the $\mathrm{Fe}-\mathrm{Cu}$ dimer after the end of the pump pulse. Between pump and probe pulse, no voltage was applied that could disturb the free evolution of the surface atom's spin. We observe $\Delta N$ to be negative and to exponentially decay to zero as $\Delta t$ is increased. $\Delta N$ is expected to be negative: In the ground state, the surface spin is aligned nearly parallel to the spin-polarization axis of the tip. Any spin excitation reduces the projection of the surface spin along the polarization axis of the tip and hence reduces the conductance of the tunnel junction. The exponential decay of $\Delta N$ is characteristic for a system with a single rate-limiting relaxation mechanism. Fitting an exponential to the time dependence of $\Delta N$ yields a relaxation time of $T_{1}=87 \pm 1 \mathrm{~ns}$.

To demonstrate that the observed exponential decay is indeed due to spin relaxation, we performed two control experiments. First, we repeated the measurement on the same $\mathrm{Fe}-\mathrm{Cu}$ dimer but with a non-spin-polarized tip (Fig. 2C). We switched between spin-polarized and non-spin-polarized STM tips by means of the reversible transfer of a $\mathrm{Mn}$ atom from the surface to the apex of the tip (15). Without spin polarization of the tip, the conductance of the tunnel junction is insensitive to the orientation of the dimer spin, and we correspondingly observe $\Delta N$ to be zero. Second, we repeated the measurement with a spin-polarized tip but on a $\mathrm{Cu}$ adatom, which has no features in its spin excitation spectrum (Fig. 2B), consistent with having no net spin. As expected, we observe $\Delta N$ to be zero in this case (Fig. 2C).
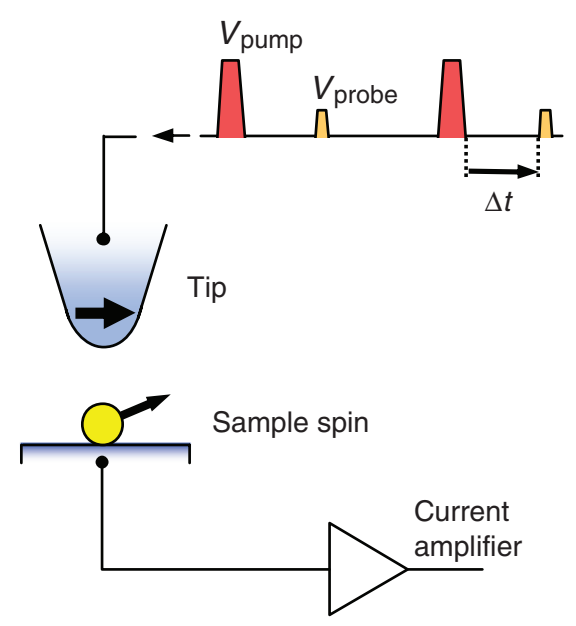

Fig. 1. Spin-sensitive pump-probe measurement scheme. The conductance of the STM tunnel junction varies according to the alignment of the sample spin with the tip spin. A series of fast voltage pulses with voltage $V_{\text {pump }}$ for the pump pulse and $V_{\text {probe }}$ for the probe pulse is sent to the tip. Measurement of the time-averaged probe-pulse current as a function of the delay time between the pulses, $\Delta t$, reveals the dynamical evolution of the sample spin. 
Fig. 2. Measurement of spin relaxation time. (A) STM topograph of an $\mathrm{Fe}-\mathrm{Cu}$ dimer and a $\mathrm{Cu}$ adatom (yellow: high; blue: low). (B) Spin excitation spectra $\mathrm{d} / / \mathrm{d} V$ versus voltage, of an $\mathrm{Fe}-\mathrm{Cu}$ dimer and a $\mathrm{Cu}$ adatom measured with a non-spin-polarized tip at magnetic field $B=7 \mathrm{~T}$. Steps in $\mathrm{d} / / \mathrm{d} V$ indicate spin excitation energies. (C) Pump-probe measurements, $\Delta N$ versus $\Delta t$, at $B=7 \mathrm{~T}$. Region I: probe pulse precedes the pump pulse; region II: pump and probe pulses overlap (fig. S1) (17); region III: probe pulse follows the pump pulse and senses the postexcitation dynamics of the system. Insets depict sample spins. For the $\mathrm{Fe}-\mathrm{Cu}$ dimer (top panel) $\Delta N$ decays exponentially in region III, with a spin relaxation time of $T_{1}=87 \pm 1 \mathrm{~ns}$ obtained from an exponential fit (magenta). Control experiments on the same $\mathrm{Fe}-\mathrm{Cu}$ dimer but without spin sensitivity in the tip (middle panel) and on a $\mathrm{Cu}$ atom with spin-sensitivity in the tip (bottom panel). the relative orientation of tip and
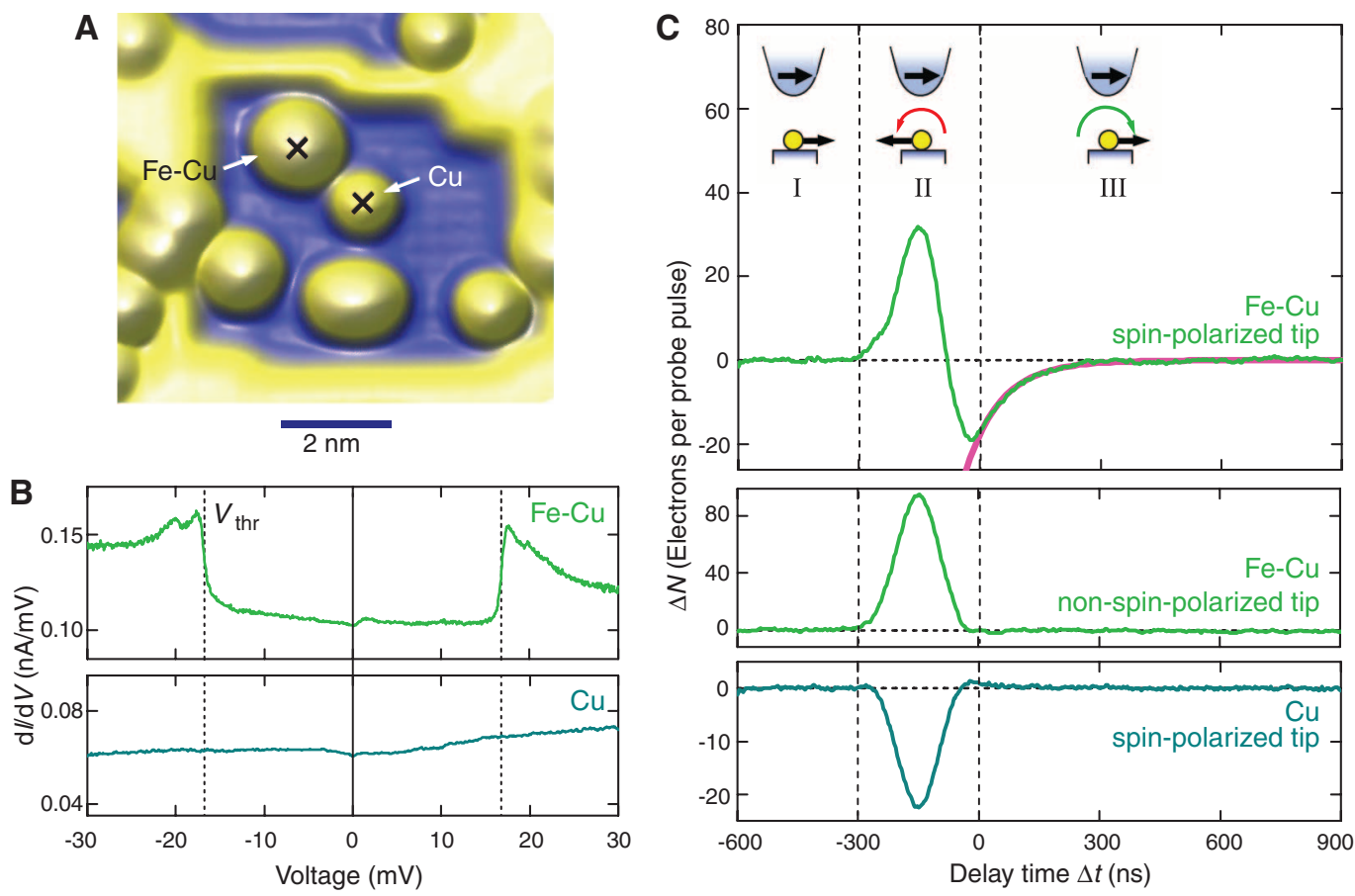

We tested whether the pump-probe measurement was itself affecting the spin relaxation time and found that $T_{1}$ does not change under variation of the amplitude and widths of the pump and probe pulses and the tip-sample separation (Fig. 3B and fig. S2). However, the magnitude of the pump-probe signal, $\Delta N$, depends strongly on the pump voltage, with a distinct onset at $V_{\text {thr }}$, below which the pump pulse does not lead to a detectable spin relaxation signal (Fig. 3A). This observation agrees with the spin excitation spectrum, where the first spin excitation is observed at the same threshold voltage (Fig. 2B). The polarity of the pump pulse is not crucial, but negative voltages help in exciting the $\mathrm{Fe}-\mathrm{Cu}$ dimer's spin by spin-momentum transfer from the spinpolarized tip (15).

The pump-probe measurements corroborate the finding of large easy-axis magnetic anisotropy for the $\mathrm{Fe}-\mathrm{Cu}$ dimers. Such anisotropy gives rise to a spin state distribution with two low-lying states, $|+2\rangle$ and $|-2\rangle$, that are separated by an energy barrier (Fig. 3C). In a magnetic field along the easy axis, $|+2\rangle$ is the ground state and $|-2\rangle$ a longlived excited state. Excitation of the $\mathrm{Fe}-\mathrm{Cu}$ dimer over the barrier starts with a transition from $|+2\rangle$ to $|+1\rangle$ and requires at least $e V_{\text {thr }}$ energy (Fig. 3C, red arrow), which results in the threshold behavior found in the pump-probe measurement (Fig. 3A). Once excited to the $|+1\rangle$ state, successive excitations and de-excitations (Fig. 3C, light red arrows) are immediately possible as none require energy exceeding $e V_{\text {thr. }}$. Upon termination of the pump pulse, the spin can be in any of the states but relaxes to either $|+2\rangle$ or $|-2\rangle$ on a time scale that is as yet too rapid for us to observe (15). The time-dependent signal recorded in the pump-
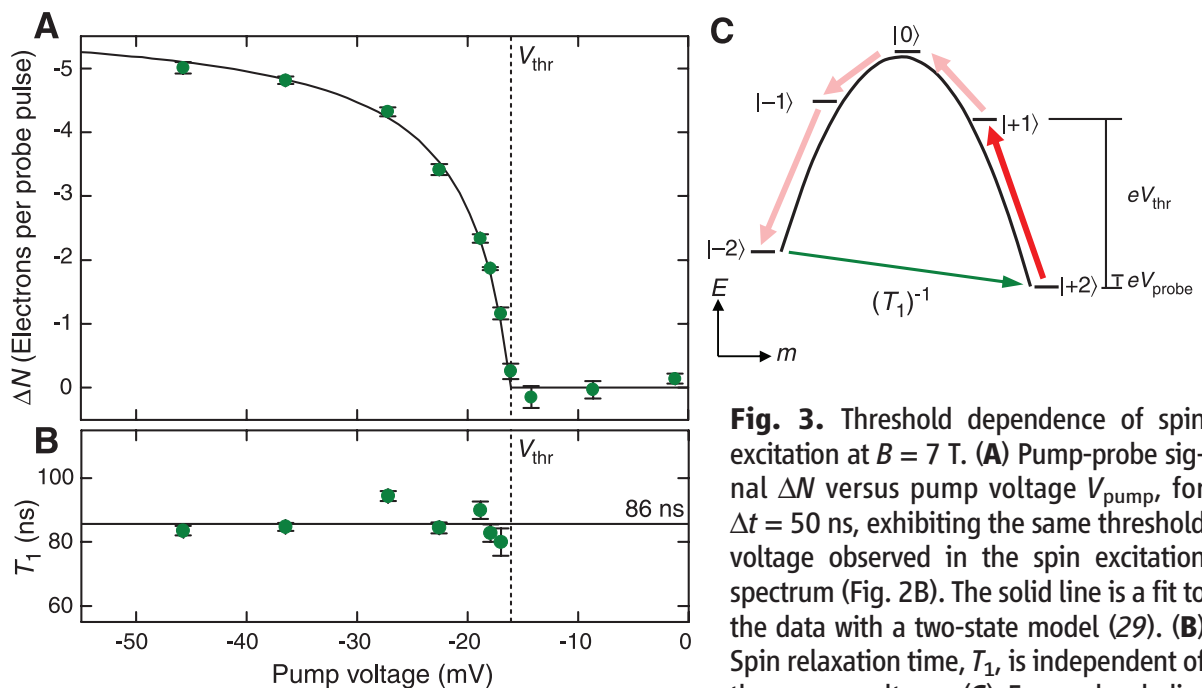

Fig. 3. Threshold dependence of spin excitation at $B=7 \mathrm{~T}$. (A) Pump-probe signal $\Delta N$ versus pump voltage $V_{\text {pump }}$, for $\Delta t=50 \mathrm{~ns}$, exhibiting the same threshold voltage observed in the spin excitation spectrum (Fig. 2B). The solid line is a fit to the data with a two-state model (29). (B) Spin relaxation time, $T_{1}$, is independent of the pump voltage. (C) Energy-level diagram of the spin states of the $\mathrm{Fe}-\mathrm{Cu}$ dimer as a function of the magnetic quantum number, $m$. The measured relaxation time, $T_{1}$, corresponds to the $|m=-2\rangle \rightarrow|+2\rangle$ transition. probe measurement for $t>0$ (Fig. 2C, region III) then corresponds to the relaxation from the longlived $|-2\rangle$ state (Fig. 3C, green arrow). At $0.6 \mathrm{~K}$ the thermal energy is insufficient to relax over the energy barrier, and indeed no thermally activated relaxation is observed for temperatures between 0.6 and $10 \mathrm{~K}$ (fig. S3). Hence, the relaxation out of $|-2\rangle$ is likely due to magnetic tunneling $(23,24)$, as we discuss next.

Figure $4 \mathrm{~A}$ shows that $\Delta N$ decays as a single exponential for all values of magnetic field, but the relaxation time varies strongly with magnetic field. $T_{1}$ first grows with magnetic field, but declines rapidly above $\sim 6 \mathrm{~T}$ (Fig. 4B). A similar non- monotonous field dependence of $T_{1}$ was recently observed for quantum tunneling of magnetization in molecular magnets (19). Finite transverse anisotropy makes magnetic tunneling possible for $\mathrm{Fe}-\mathrm{Cu}$ dimers by mixing the $|-2\rangle$ and $|+2\rangle$ states. As the magnetic field is increased from zero, the Zeeman energy increasingly splits these states, which reduces the tunneling matrix element between them and thereby increases $T_{1}$ (23). The reduction in $T_{1}$ above $6 \mathrm{~T}$ indicates a nonzero angle between the magnetic field and the easy magnetic axis of the $\mathrm{Fe}-\mathrm{Cu}$ dimer. A transverse component of the magnetic field increases mixing of the spin states and shortens $T_{1}(25)$. 

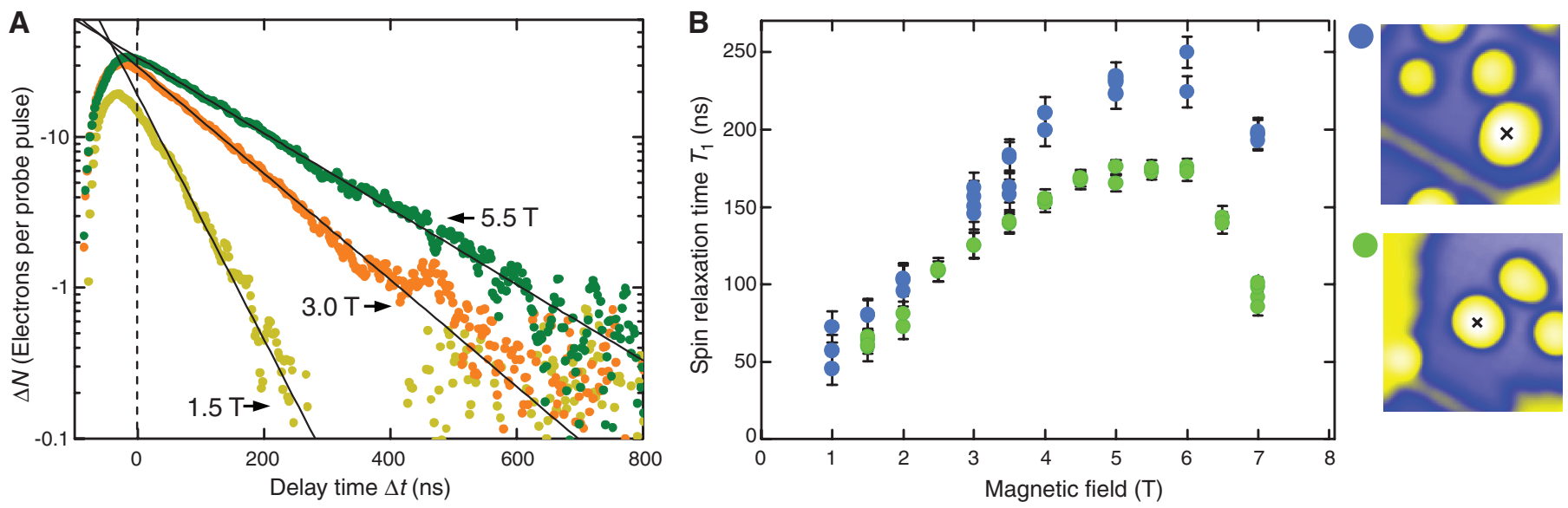

Fig. 4. Field and site dependence of the spin relaxation time. (A) Pump-probe measurements for different magnetic fields on an Fe-Cu dimer; solid lines are exponential fits. (B) $T_{1}$ as a function of magnetic field for the two Fe-Cu dimers shown in the accompanying 5-nm by 5-nm STM topographs.

One of the Fe-Cu dimers in Fig. 4B always exhibits a larger $T_{1}$ than the other. We speculate that this variation is due to differences in the nearby surface features as seen in the accompanying topographs. This observation emphasizes the capability of the all-electronic pump-probe technique presented here to resolve local variations in the spin relaxation time with atomic precision.

The pump-probe scheme we have described can be used to monitor the temporal evolution of any excitation provided (i) the excitation can be driven by tunneling electrons; (ii) the conductance of the tunnel junction exhibits a postexcitation time dependence; and (iii) the system evolves on an accessible time scale. Excitations fulfilling these requirements include long-lived vibrational excitations, conformational changes of molecules (26) such as in molecular motors (27), or fast localized heating (28). We emphasize that this pump-probe scheme can in principle be used to monitor the dynamical evolution of the excited state, not just its relaxation; with sufficient temporal resolution it should be possible to monitor the vibration of an atom or molecule and even the precession of a spin.

\section{References and Notes}

1. C. P. Slichter, Principles of Magnetic Resonance (Springer, Berlin and New York, 1996).

2. D. Rugar, R. Budakian, H. J. Mamin, B. W. Chui, Nature 430, 329 (2004).

3. J. R. Maze et al., Nature 455, 644 (2008).

4. G. Balasubramanian et al., Nature 455, 648 (2008)

5. M. Bode et al., Nature 447, 190 (2007).

6. A. J. Heinrich, J. A. Gupta, C. P. Lutz, D. M. Eigler, Science 306, 466 (2004).

7. N. Tsukahara et al., Phys. Rev. Lett. 102, 167203 (2009).

8. C. F. Hirjibehedin et al., Science 317, 1199 (2007).

9. X. Chen et al., Phys. Rev. Lett. 101, 197208 (2008).

10. C. F. Hirjibehedin, C. P. Lutz, A. J. Heinrich, Science 312, 1021 (2006).

11. M. J. Rost et al., Rev. Sci. Instrum. 76, 053710 (2005)

12. C. Durkan, M. E. Welland, Appl. Phys. Lett. 80, 458 (2002).

13. G. Nunes Jr., M. R. Freeman, Science 262, 1029 (1993).

14. O. Takeuchi et al., Appl. Phys. Lett. 85, 3268 (2004).

15. S. Loth et al., Nat. Phys. 6, 340 (2010).

16. R. Wiesendanger, Rev. Mod. Phys. 81, 1495 (2009).

17. Materials and methods are available as supporting material on Science Online.

18. M. N. Leuenberger, D. Loss, Nature 410, 789 (2001).

19. D. E. Freedman et al., J. Am. Chem. Soc. 132, 1224 (2010).

20. A. Ardavan et al., Phys. Rev. Lett. 98, 057201 (2007)
21. T. Choi, C. D. Ruggiero, ]. A. Gupta, Phys. Rev. B 78, 035430 (2008).

22. F. Komori, S.-Y. Ohno, K. Nakatsuji, Prog. Surf. Sci. 77 1 (2004).

23. D. Gatteschi, R. Sessoli, ]. Villain, Molecular Nanomagnets (Oxford Univ. Press, New York, 2006).

24. C. Sangregorio, T. Ohm, C. Paulsen, R. Sessoli, D. Gatteschi, Phys. Rev. Lett. 78, 4645 (1997)

25. W. Wernsdorfer, R. Sessoli, Science 284, 133 (1999).

26. K. Henzler-Wildman, D. Kern, Nature 450, 964 (2007).

27. W. R. Browne, B. L. Feringa, Nat. Nanotechnol. 1, 25 (2006).

28. B. Barwick, H. S. Park, O.-H. Kwon, ]. S. Baskin, A. H. Zewail, Science 322, 1227 (2008)

29. J. A. Gupta, C. P. Lutz, A. ]. Heinrich, D. M. Eigler, Phys. Rev. B 71, 115416 (2005).

30. We thank B. Melior for technical assistance. S.L., C.P.L., and A.].H. acknowledge financial support from the Office of Naval Research, S.L. from the Alexander von Humboldt Foundation, and M.E. from European Science Foundation project FunSMARTs II.

\section{Supporting Online Material}

www.sciencemag.org/cgi/content/full/329/5999/1628/DC1

Materials and Methods

Figs. S1 to S3

References

Movie S1

30 April 2010; accepted 4 August 2010

$10.1126 /$ science.119168

\section{Optical Clocks and Relativity}

\section{W. Chou, * D. B. Hume, T. Rosenband, D. J. Wineland}

Observers in relative motion or at different gravitational potentials measure disparate clock rates. These predictions of relativity have previously been observed with atomic clocks at high velocities and with large changes in elevation. We observed time dilation from relative speeds of less than 10 meters per second by comparing two optical atomic clocks connected by a 75-meter length of optical fiber. We can now also detect time dilation due to a change in height near Earth's surface of less than 1 meter. This technique may be extended to the field of geodesy, with applications in geophysics and hydrology as well as in space-based tests of fundamental physics.

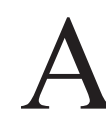
lbert Einstein's theory of relativity forced us to alter our concepts of reality. One of the more startling outcomes of the theory is that we have to give up our notions of simul- taneity. This is manifest in the so-called twin paradox (1), in which a twin sibling who travels on a fast-moving rocket ship returns home younger than the other twin. This "time dilation" can be quantified by comparing the tick rates of identical clocks that accompany the traveler and the stationary observer. Another consequence of Einstein's theory is that clocks run more slowly near massive objects. In the range of speeds and length scales encountered in our daily life, relativistic effects are extremely small. For example, if two identical clocks are separated vertically by $1 \mathrm{~km}$ near the surface of Earth, the higher clock emits about three more second-ticks than the lower one in a million years. These effects of relativistic time dilation have been verified in several important experiments $(2-6)$

Time and Frequency Division, National Institute of Standards and Technology (NIST), Boulder, CO 80305, USA.

*To whom correspondence should be addressed. E-mail: chinwen@nist.gov 